\title{
VLBI telescopes' gravitational deformations investigated with terrestrial surveying methods
}

\section{P. Sarti* ${ }^{*}$ C. Abbondanza $\cdot$ M. Negusini}

Istituto di Radioastronomia (IRA)- Istituto Nazionale di Astrofisica (INAF)

Via P. Gobetti, 10140129 Bologna, Italy

Tel.: +39-051-6399417

Fax: +39-051-6399431

E-mail: p.sarti@ira.inaf.it

E-mail: c.abbondanza@ira.inaf.it

E-mail: m.negusini@ira.inaf.it

\section{Vittuari}

DISTART, Area Geodesia e Topografia - Universitá di Bologna

Viale Risorgimento, 240136 Bologna, Italy

E-mail: luca.vittuari@mail.ing.unibo.it

Large VLBI telescopes undergo gravitational deformations which affect both geodetic and astronomic observations. In order to assess the extent and magnitude of such deformations and to evaluate their effect on telescopes' performances, terrestrial surveying methods can be applied to monitor the telescopes' structure at different pointing elevations. Finite Element Model analysis, laser scanner surveying, trilateration and triangulation have been applied on the telescope in Medicina to estimate i) the deformations of the primary mirror and to monitor ii) the position of the feed horn located at the primary focus and iii) the position of the vertex of the paraboloid. If detectable, these deformations modify the position of the primary focus and the signal path length and may therefore reduce the antenna gain and bias the phase of the incoming signal. We are presenting the investigations performed on the Medicina VLBI telescope, quantifying the magnitude of the deformations of the primary dish, the quadrupode and the vertex and we are also presenting an elevation dependent model for signal path corrections.

The 9th European VLBI Network Symposium on The role of VLBI in the Golden Age for Radio Astronomy and EVN Users Meeting

September 23-26, 2008

Bologna, Italy

\footnotetext{
* Speaker.
} 


\section{Introduction}

Indirect methods applied in local tie surveying are a flexible tool for retrieving further information than the mere eccentricity vector estimate. The analysis of the circular paths described by the targets (e.g. retro reflecting prisms) that are installed on the telescope's structure and surveyed from the local ground control network may be useful in highlighting the parts of the telescope that are remarkably affected and deformed by gravity.

Gravitational deformations are elevation dependent; they may affect the path length of the incoming signal and may bias its phase, thus corrupting the quality of the data acquired by the telescope. Gravitational deformations depend on the pointing elevation of the telescope and an attempt to model their impact on the VLBI observable was successfully pursued by [1], with the use of a FEM (Finite Element Model). Their paper is a comprehensive discussion of the theoretical aspects related to deformations of VLBI telescopes and a remarkable example of FEM application aimed at solving the problem of signal path variations in the VLBI antenna in Fairbanks (Alaska, USA). According to [1], the signal path variations can be described as the linear combination of three terms:

$$
\Delta L=\alpha_{R} \Delta R+\alpha_{V} \Delta V+\alpha_{F} \Delta F
$$

where $\Delta R$ is the component along the line of sight of the displacement of the receiver, $\Delta V$ the displacement, along the line of sight, of the primary mirror (essentially described by its vertex's position) and $\Delta F$ the change of focal length. The linear coefficients $\alpha_{R}, \alpha_{V}$ and $\alpha_{F}$ depend on the dimensions and structure of the telescope [1]. In order to quantify $\Delta L$, we have consistently applied independent methods: terrestrial surveying (particularly triangulation and trilateration), laser scanning and FEM. A combination of these methods was applied to investigate the deformations of the telescope in Medicina, with the specific purpose of cross checking the results obtained on some particular parts of the telescope and confirm the overall deformation pattern depicted by the analysis. A detailed discussion of the surveying strategy and the analysis procedure adopted to compute the signal path variation $\Delta L$ for the telescope in Medicina is contained in [2].

\section{Terrestrial techniques}

Laser scanning on one side and triangulation and trilateration on the other are terrestrial surveying approaches that proved to be effective in determining the focal length variation (i.e. the deformation of the main reflector's surface) and the motion of the receiver. The FEM was successfully applied to determine the motion of the vertex of the paraboloid and the motion of the receiver; this latter was therefore determined with two different methods and the deformation experienced by the quadripod could be cross-checked and verified. The agreement of results obtained with the two independent methods was excellent.

The deformations of the main reflector determined with the laser scanner are shown in figure (1) as surveyed at six telescope's pointing elevation $(15,30,45,60,75$ and 90) deg. As the elevation changes from high to low elevations, the dish, quite regularly, folds in under the varying effect of gravity: at zenith the surface of the paraboloid is pulled down towards the ground and is therefore more widely open. The consequence is an increment of the value of the focal length of the paraboloid (see figure 2) which was determined with a least squares approach on the cloud points 
Figure 1: Differences between the laser scanned surface at different elevations and the theoretical paraboloid $(f=10.2590 \mathrm{~m})$. Cold colors correspond to smaller differences; warm colors to higher ones. The deformations reach their maximum value of approximately $2 \mathrm{~cm}$ at the edge of the dish.
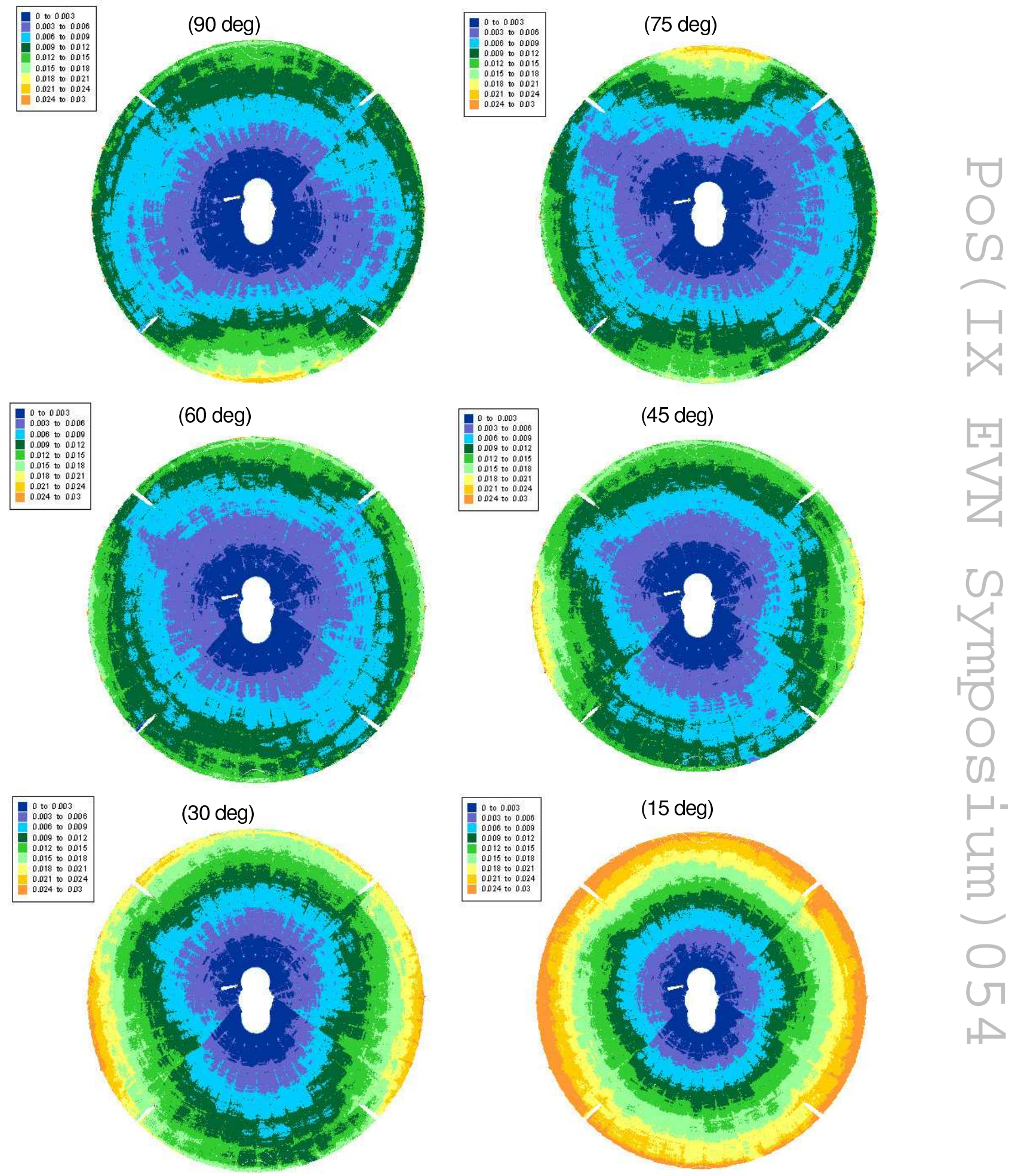
Figure 2: Behaviour of the focal length as the elevation varies: the greatest value is found at zenith, when gravity acts on the dish slightly flattening it out towards the ground.

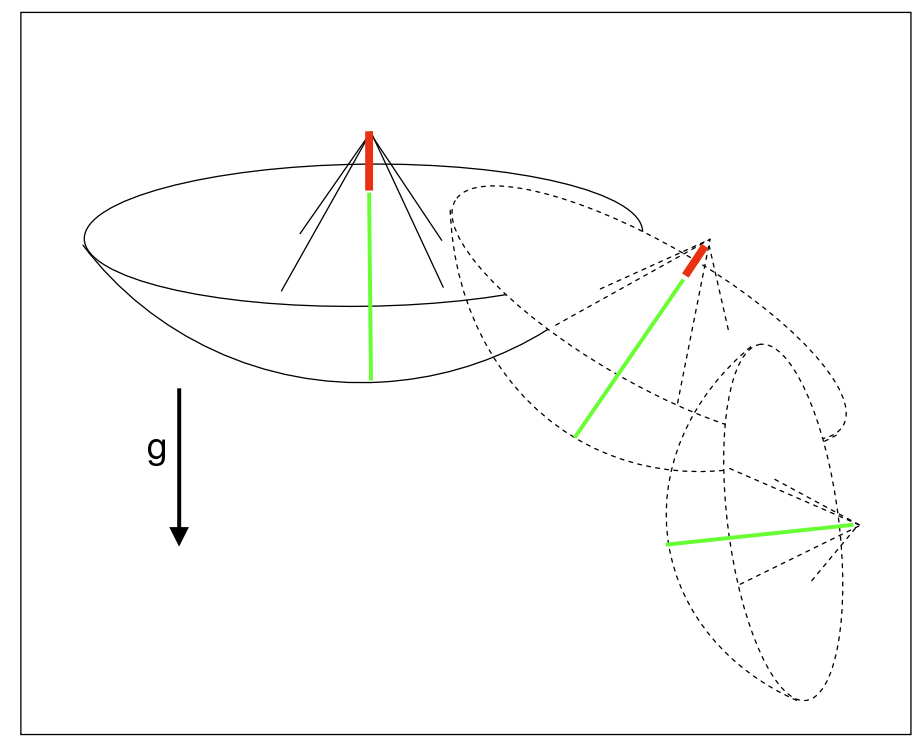

[3]; the estimated focal length variations are shown in figure (3).

Figure 3: Experimental values of $\Delta R$ (blue circles), $\Delta V$ (red triangles), $\Delta F$ (green squares) and the corresponding interpolating second order functions. In black is represented the $\Delta L$ obtained according to equation (1.1).

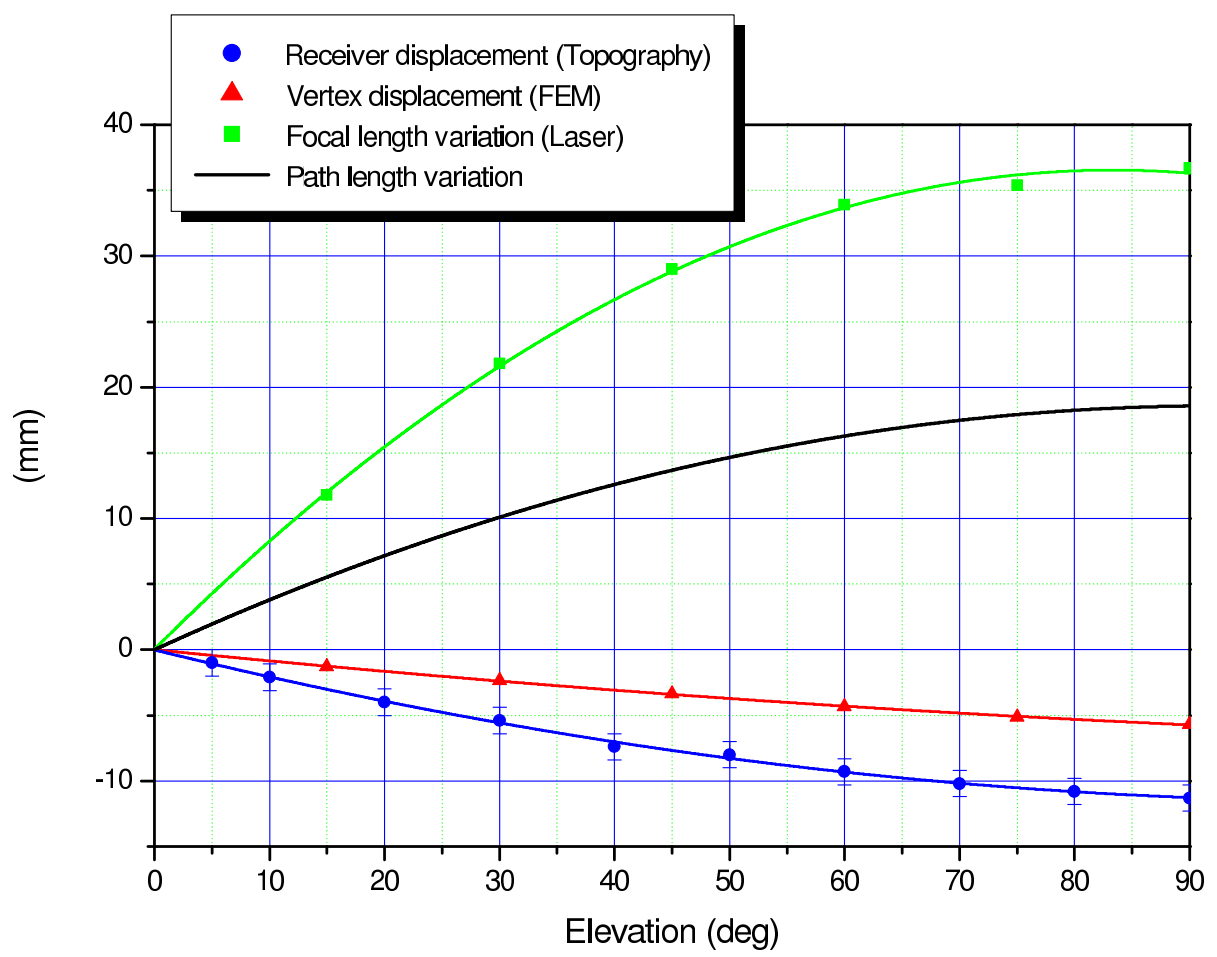


A complete description of the compound process of laser scanning surveying and data processing can be found in [3].

Gravity acts similarly on the vertex and on the receiver. Ideally, as the antenna is steered in elevation, the points should follow an arc of a circle whose centre belongs to the elevation axis. At zenith, gravity deforms the structure pulling downward both points and therefore diminishing their distance from the elevation axis; as a consequence, the arc is flattened out (see figure (4)). The

Figure 4: Ideal circular path described by the vertex or the receiver (black dashed line) and real deformed path under the effect of gravity (dashed red line). The displacement to be determined $\Delta V$ or $\Delta R$ is the distance between the two curves computed along the radius. The rotation centre is given by the elevation axis.

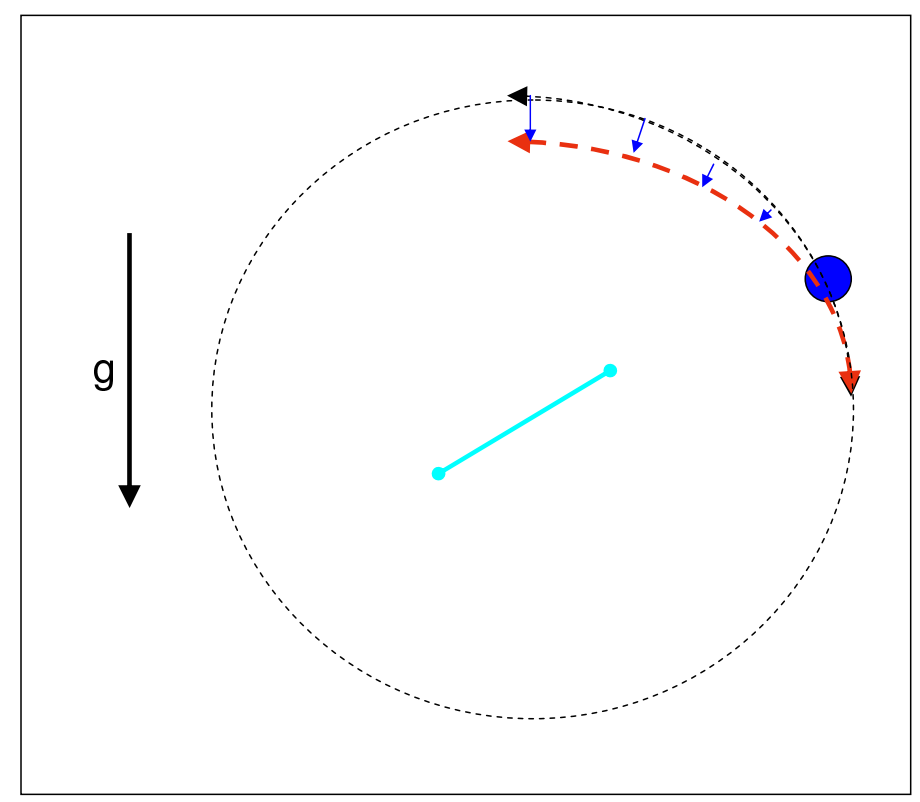

component, along the line of sight, of the decrease of the relative distance vertex-elevation axis was determined with FEM and is reported in figure (3). Analogously, the variation of the distance receiver-elevation axis (along the same direction) was determined with FEM and terrestrial triangulation and trilateration; this latter determination is reported in the same figure (3).

\section{Results and conclusions}

The linear combination, according to equation (1.1), of the variations of $\Delta V, \Delta R$ and $\Delta F$, gives the variation of the signal path length $\Delta L$ shown with a black line in figure (3). $\Delta L$ varies up to $2 \mathrm{~cm}$ in the elevation range $[0,90]$ deg and causes remarkable effects on the phase of the incoming signal. Nevertheless, the combination of terrestrial triangulation and trilateration, laser scanning and FEM efficiently solves the problem and can be applied to correct the phase biases due to gravitational deformations.

\section{References}

[1] Clark, T.A. and Thomsen, P. (1988) Deformations in VLBI antennas. Tech. rep., 100696, NASA, Greenbelt, MD 
[2] Sarti, P., Abbondanza, C. and Vittuari, L. (2009) Gravity dependent signal path variation in a large VLBI telescope modelled with a combination of surveying methods. Submitted to J Geodesy

[3] Sarti, P., Vittuari, L. and Abbondanza, C. (2009) Laser scanner and terrestrial surveying applied to gravitational deformation monitoring of large VLBI telescopes' primary reflector. in press J Surv Eng 